\title{
Observation of Negative Differential Conductance in a Reverse-Biased Ni/Ge Schottky Diode
}

\author{
Muhammad Khaled Husain, Student Member, IEEE, Xiaoli V. Li, Member, IEEE, and \\ Cornelis H. de Groot, Senior Member, IEEE
}

\begin{abstract}
In this letter, we report the experimental observation of negative differential conductance (NDC) in a Ni/Ge Schottky diode. With the aid of theoretical models and numerical simulation, we show that, at reverse bias, electrons tunnel into the high electric field of the depletion region. This scatters the electrons into the upper valley of the Ge conduction band, which has a lower mobility. The observed NDC is hence attributed to the transferred-electron effect. This shows that Schottky contacts can be used to create hot electrons for transferred-electron devices.
\end{abstract}

Index Terms-Electrodeposition, germanium, Gunn diode, Schottky barrier (SB), transferred-electron effect.

\section{INTRODUCTION}

$\mathbf{T}$ HE TRANSFERRED-ELECTRON effect leads to negative differential resistivity in semiconductors having twovalley conduction-band profile, e.g., in GaAs [1], InP [2], and Ge [3]. The corresponding device, known as the transferredelectron device, has been extensively used in microwave circuits. Injection-limited cathode contacts, in particular, graded $\mathrm{Al}_{x} \mathrm{Ga}_{1-x} \mathrm{As}$, have been used [4] instead of ohmic contacts in GaAs-based devices to improve device performance. The extreme Fermi-level pinning in semiconductors hampers the use of thermionic emission (TE) over a Schottky barrier (SB) for hot electron injection [5]. In this letter, we report the first experimental observation of negative differential conductance (NDC) in a reverse-biased $\mathrm{Ni} / \mathrm{Ge}$ diode at low temperature, and we show that thermionic field emission (TFE) through the Schottky diode is the mechanism.

\section{EXPERIMENTS AND RESULTS}

$\mathrm{Ni} / \mathrm{n}+\mathrm{Ge}$ contacts were fabricated on $400-\mu \mathrm{m}$-thick n-doped Ge (100) wafers with a resistivity of $0.005-0.02 \Omega \cdot \mathrm{cm}$. For comparison, $\mathrm{Ni} / \mathrm{nGe}$ contacts were also fabricated where $\mathrm{Ge}$ resistivity was $2-2.4 \Omega \cdot \mathrm{cm}$. Square Ni pads with $400-\mu \mathrm{m}$ sides were fabricated using electrodeposition. Details on the fabrication and measurement can be found in our recent work [6]. The low-temperature measurements down to $50 \mathrm{~K}$ were performed using a Bio-Rad DL 4960 cryostat.

Manuscript received May 16, 2009. First published July 31, 2009; current version published August 27, 2009. The review of this letter was arranged by Editor C. Bulucea.

The authors are with the Nano Research Group, School of Electronics and Computer Science, University of Southampton, SO17 1BJ Southampton, U.K. (e-mail: mkh05r@ecs.soton.ac.uk; x104r@ecs.soton.ac.uk; chdg@ecs. soton.ac.uk).

Color versions of one or more of the figures in this letter are available online at http://ieeexplore.iee.org.

Digital Object Identifier 10.1109/LED.2009.2025673

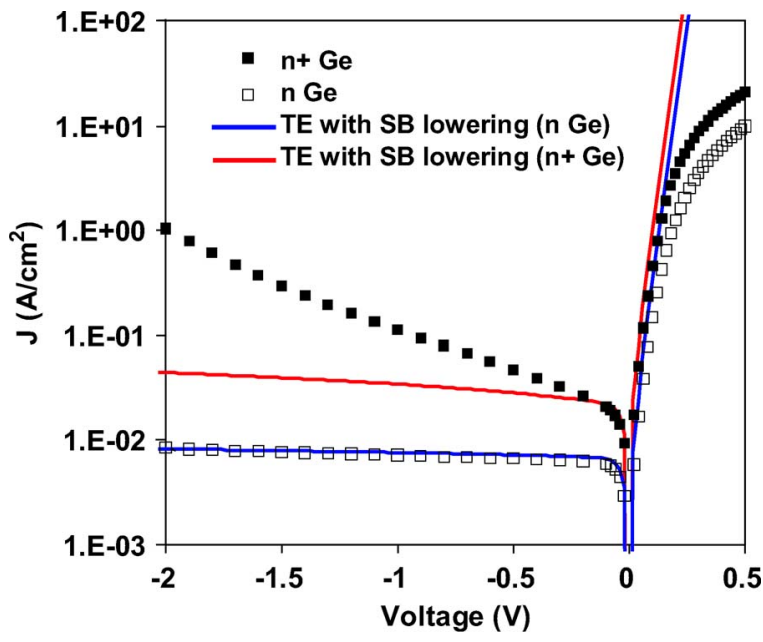

Fig. 1. Room-temperature $J-V$ characteristics of electrodeposited $\mathrm{Ni} / \mathrm{n}+\mathrm{Ge}$ and $\mathrm{Ni} / \mathrm{nGe}$ contacts. Theoretical models using the SB height extracted from the forward-bias characteristic are also shown.

Room-temperature current density $(J)$ versus voltage $(V)$ characteristics of a $\mathrm{Ni} / \mathrm{n}+\mathrm{Ge}$ contact are shown in Fig. 1. For comparison, the $J-V$ curve of a $\mathrm{Ni} / \mathrm{nGe}$ contact is presented in which TE is the dominant transport mechanism. By extrapolation from the exponential forward-bias region, an SB height $\phi$ of $0.53 \mathrm{eV}$ is obtained. Taking the image-force lowering and the substrate doping density $N_{d}$ of $8.7 \times 10^{14} \mathrm{~cm}^{-3}$ (obtained by $C-V$ method [6]) into account, the theoretical fitting characteristic is shown as a solid line in Fig. 1 . For the $\mathrm{Ni} / \mathrm{n}+\mathrm{Ge}$ contact, excess current is evident in the reverse bias. The theoretical TE characteristic of this diode considering barrier lowering was calculated using a substrate doping density of $2.9 \times 10^{17} \mathrm{~cm}^{-3}$ (obtained by $C-V$ method) and a barrier height of $0.52 \mathrm{eV}$ (obtained by $J-V$ method). Although a significant increase in current is caused by image-force lowering, it is not enough to explain the excess currents for $n+G e$. The earlier considerations suggest TFE as the dominant transport mechanism at electrodeposited $\mathrm{Ni} / \mathrm{n}+\mathrm{Ge}$ contacts.

In order to investigate the TFE of the $\mathrm{Ni} / \mathrm{n}+\mathrm{Ge}$ contact at low temperatures $(T), I-V$ measurements were performed down to $50 \mathrm{~K}$. For clarity, only five curves are shown in Fig. 2(a). As the temperature was reduced, the change in the saturation current in the forward bias was insignificant. This is in agreement with the TFE model for a highly doped semiconductor [7]. However, in the reverse bias, an unusual current-conduction mechanism at low temperature is observed. At biases in the range of $-1-$ $-1.5 \mathrm{~V}$, the effect of NDC is revealed for $T \leq 200 \mathrm{~K}$. From the 

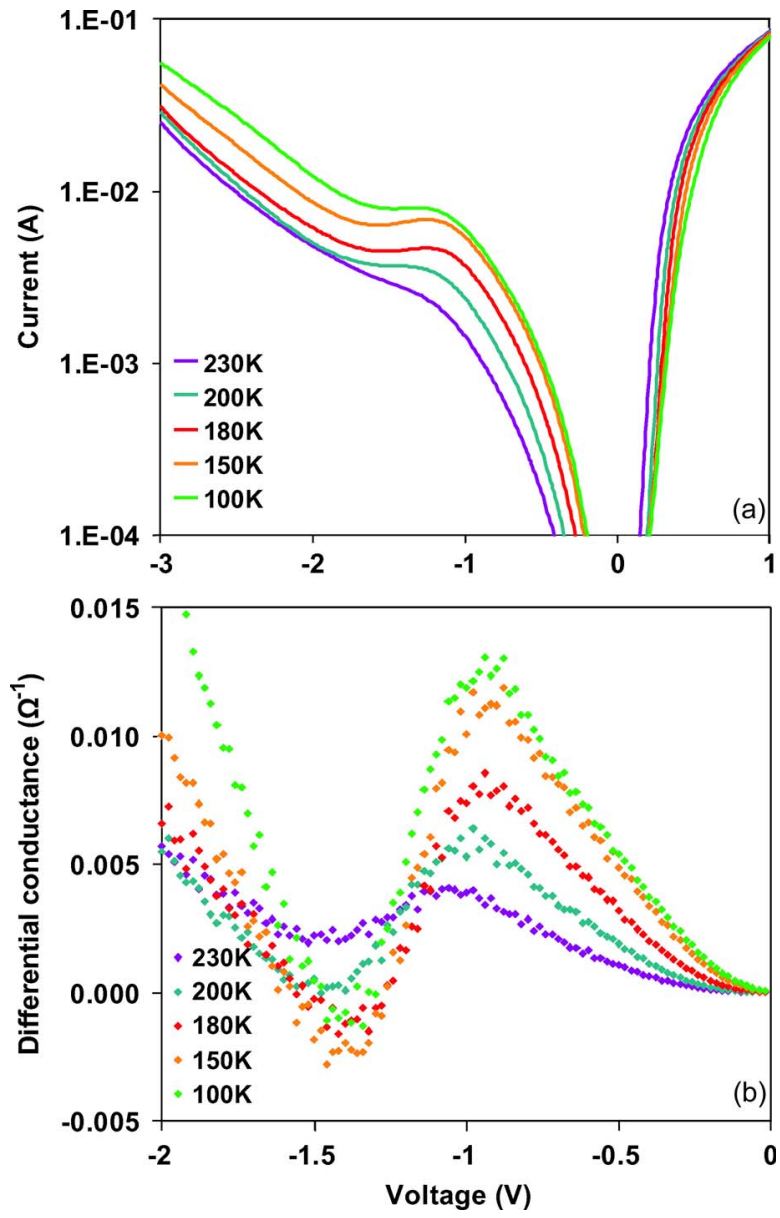

Fig. 2. (a) $I-V$ characteristics measured at low temperatures for the electrodeposited $\mathrm{Ni} / \mathrm{n}+\mathrm{Ge}$ diode with a contact area of $400 \mu \mathrm{m}$ square. (b) Differential conductance as a function of voltage for various $T$.

$I-V$ curves shown in Fig. 2(a), the differential conductance was calculated for each bias voltage and is shown as a function of $T$ in Fig. 2(b). A small but clear indication of NDC is observed at $T \leq 200 \mathrm{~K}$ and at reverse-bias voltage of $\sim 1.5 \mathrm{~V}$.

To exclude a bulk NDC effect, we investigated the application of similar applied biases $(\sim 1.5 \mathrm{~V})$ in a bulk Ge substrate as in the $\mathrm{Ni} / \mathrm{Ge}$ diodes. Back-to-back ohmic contacts of $\mathrm{Au}-\mathrm{Sb}$ were deposited on the Ge substrates followed by annealing under the same condition as for ohmic contacts of the Schottky devices. Electrical measurements at low temperatures (results not shown) revealed the absence of any NDC in the bulk Ge.

\section{Discussions}

We attribute the observed NDC in our device to an intervalley or intersubband electron transfer to a low-mobility band in Ge. The intersubband spacing (shown schematically in Fig. 3) between the $L$-point and the $X$-point minima of the Ge conduction band is $0.18 \mathrm{eV}[8]$. The $X$-point minimum is characterized by a lower mobility (heavier effective mass). Therefore, any scattering to this minimum would exhibit NDC. To gain enough energy for this transfer, a high electric field is required, which, in our case, is supplied by the depletion region of the $\mathrm{Ni} / \mathrm{n}+\mathrm{Ge}$ $\mathrm{SB}$ in reverse bias. The control sample did not show any NDC due to the applied field $(\sim 37 \mathrm{~V} / \mathrm{cm})$ being much lower than the

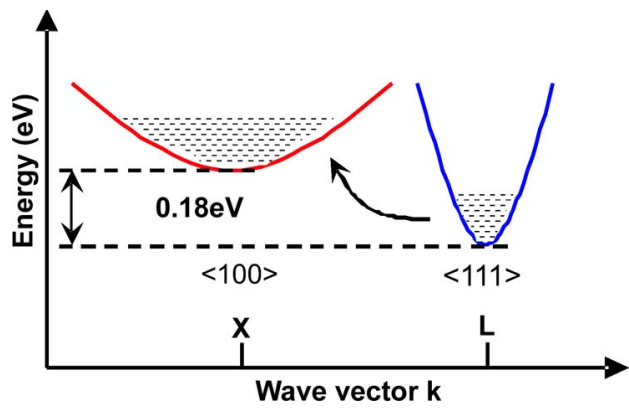

Fig. 3. Schematic representation of the conduction band of Ge showing the minima at the $X$ (high energy, low mobility) and the $L$ (low energy, high mobility) band.

threshold field ( $2.3 \mathrm{kV} / \mathrm{cm}$ at $77 \mathrm{~K}$ [9]) for a transferred-electron effect in Ge. We also note that NDC has been observed in SBbased MOSFETs due to the existence of gate-oxide charge traps [10], [11] but this explanation is not relevant to our device.

To determine the excess energy of the tunneling electrons in the reverse-biased $\mathrm{SB}$, the distribution of electrons is calculated. This distribution is given by the product of the probability $(P)$ of a triangular barrier being penetrated by an electron with energy above the metal Fermi level $(E)$ and the probability of finding an electron at that energy. The tunneling probability $P$, calculated assuming the WKB approximation, is given by [12]

$$
P(E)=\exp \left\{\frac{-\frac{2}{3}(\phi-E)^{3 / 2}}{E_{00}(\phi-V-\xi)^{1 / 2}}\right\}
$$

where $\xi$ is the depth of the Fermi level below the conduction band and $\mathrm{E}_{00}$ is given by

$$
E_{00}=\frac{\hbar}{2} \sqrt{\frac{N_{d}}{m^{*} \epsilon}}
$$

where $m^{*}$ is the effective mass of electrons and $\epsilon$ is the static dielectric constant of semiconductors. The electron distribution is given by the Fermi equation

$$
F(E)=\frac{1}{1+\exp (E / k T)}
$$

where $k$ is the Boltzmann constant. The peak of the distribution given by the product of $P(E)$ and $F(E)$, at various bias voltages and temperatures, gives the most probable energy $\left(E_{m}\right)$ of tunneling electrons. The obtained values of $E_{m}$ are shown in Fig. 4 as a function of temperature for various reverse biases. A relative transverse effective mass of 0.08 [8] for $\langle 111\rangle$-valley conduction electrons in Ge was used for the calculations. At the reverse bias of $1.25 \mathrm{~V}$, for example, the peak energy $E_{m}$ occurs at $\sim 0.18$ and $\sim 0.04 \mathrm{eV}$ for temperatures of 230 and $180 \mathrm{~K}$, respectively. These distribution functions are shown schematically in Fig. 5.

The conduction band at the depletion region in a reversebiased $\mathrm{Ni} / \mathrm{n}+\mathrm{Ge}$ Schottky diode was calculated using the commercial TCAD simulator Sentaurus Device. The effect of image-force barrier lowering and band-gap narrowing has been taken into account. The calculated conduction-band energy in the $\mathrm{Ni} / \mathrm{Ge} \mathrm{SB}$ at a reverse bias of $1.25 \mathrm{~V}$ is shown in Fig. 5. 


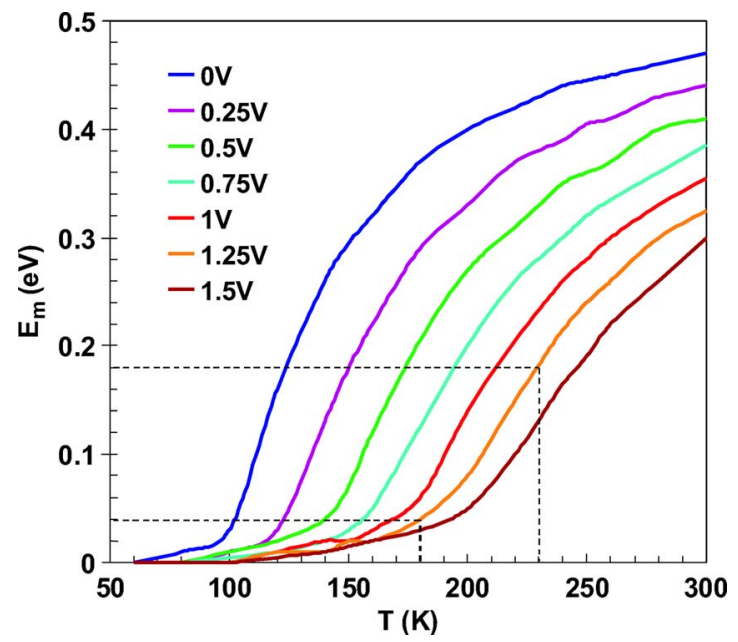

Fig. 4. Calculated most probable energy $\left(E_{m}\right)$ of tunneling electrons versus $T$ for various reverse-bias voltages applied to the $\mathrm{Ni} / \mathrm{Ge}$ Schottky diode.

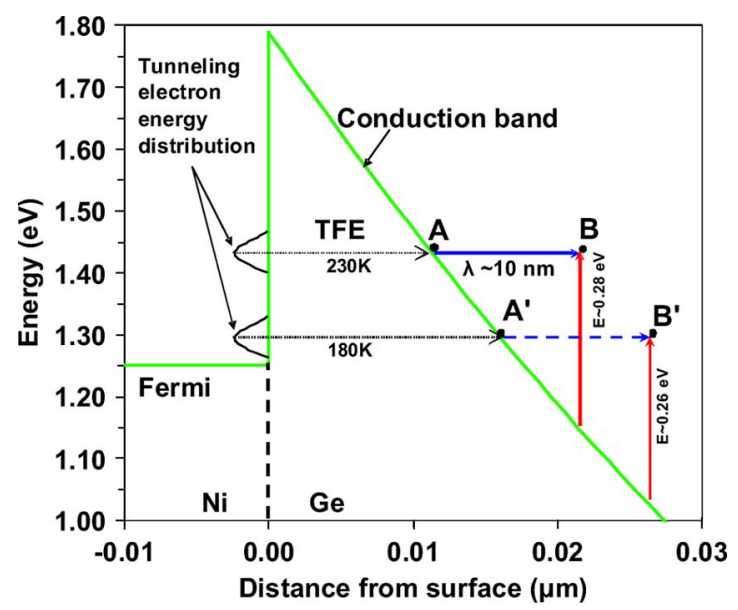

Fig. 5. Conduction-band profile obtained by numerical simulation of a $\mathrm{Ni} / \mathrm{Ge}$ Schottky diode at a reverse bias of $1.25 \mathrm{~V}$.

Following our previous calculation, TFE occurs at an energy of $1.43 \mathrm{eV}(1.25+0.18)$ at $230 \mathrm{~K}$. Upon tunneling, the injected electrons (represented by point A in Fig. 5) will be thermalized after traveling a distance of a mean free path $\lambda$ and belong to either the upper valley or the lower valley depending upon the energy of electrons. As an approximate estimation, we assume for this distance a phonon mean free path $(\simeq 105 \AA)$ [9]. The injected electrons experiences a depletion field $\mathcal{E}$ of $2.8 \times$ $10^{5} \mathrm{~V} / \mathrm{cm}$ at point A. Therefore, electrons of most probable energy, which tunnel at an energy level of $1.43 \mathrm{eV}$, will have an energy of $\lambda \mathcal{E}=0.28 \mathrm{eV}$ upon thermalization (point $\mathrm{B}$ ). Similarly, electrons tunneling at $180 \mathrm{~K}$ (point $\mathrm{A}^{\prime}$ ) will have an energy of $0.26 \mathrm{eV}$ upon thermalization (point $\mathrm{B}^{\prime}$ ). These hot electrons could easily undergo intervalley scattering of $0.18 \mathrm{eV}$ and thereby exhibiting NDC in the Ni/Ge Schottky diode. The electric field in the bulk $\mathrm{n}+$ region at $1.5-\mathrm{V}$ reverse bias, calculated to be $\sim 5 \times 10^{-5} \mathrm{~V} / \mathrm{cm}$, is low enough to exclude the onset of transferred electron effect from the bulk. The limits of NDC, at reverse biases larger than $2 \mathrm{~V}$, as shown in Fig. 2(a), is related to the population of carrier in the lower valley, as explained in literature [13].

At NDC, the $\mathrm{Ni} / \mathrm{n}+\mathrm{Ge}$ diodes show current density of $\sim 1-$ $5 \mathrm{~A} / \mathrm{cm}^{2}$ [from Fig. 2(a)]. This needs to be many orders of magnitude higher to be useful for Gunn device applications. One way to increase the reverse-bias current density could be the growth of a thin $(\sim 2 \mathrm{~nm})$ heavily n-doped $\left(1 \times 10^{20} \mathrm{~cm}^{-3}\right)$ Ge layer at the $\mathrm{Ni} / \mathrm{Ge}$ interface. Our simulations show that this could increase the current density by up to five orders in magnitude. The results presented in this letter hence could have an impact on the design of Gunn diodes.

\section{ACKNOWLEDGMENT}

The authors would like to thank C. Morrison, D. Wang, and P. de Groot of the School of Physics and Astronomy, University of Southampton, for their support in the low-temperature measurements.

\section{REFERENCES}

[1] B. K. Ridley, "Anatomy of the transferred-electron effect in III-V semiconductors," J. Appl. Phys., vol. 48, no. 2, pp. 754-764, Feb. 1977.

[2] H. Eisele, "InP Gunn devices for 400-425 GHz," Electron. Lett., vol. 42, no. 6, pp. 358-359, Mar. 2006.

[3] D. M. Chang and J. G. Ruch, "Measurement of the velocity field characteristic of electrons in germanium," Appl. Phys. Lett., vol. 12, no. 3, pp. 111-112, Feb. 1968.

[4] A. Forster, M. Lepsa, D. Freundt, J. Stock, and S. Montanari, "Hot electron injector Gunn diode for advanced driver assistance systems," Appl. Phys. A, Mater. Sci. Process., vol. 87, no. 3, pp. 545-558, Jun. 2007.

[5] W. E. Spicer, I. Lindau, P. Skeath, C. Y. Su, and P. Chye, "Unified mechanism for Schottky-barrier formation and III-V oxide interface states," Phys. Rev. Lett., vol. 44, no. 6, pp. 420-423, Feb. 1980.

[6] M. K. Husain, X. V. Li, and C. H. de Groot, "High-quality Schottky contacts for limiting leakage currents in Ge-based Schottky barrier MOSFETs," IEEE Trans. Electron Devices, vol. 56, no. 3, pp. 499-504, Mar. 2009.

[7] M. Kiziroglou, X. Li, A. Zhukov, P. de Groot, and C. de Groot, "Thermionic field emission at electrodeposited Ni-Si Schottky barriers," Solid State Electron., vol. 52, no. 7, pp. 1032-1038, Jul. 2008.

[8] W. Fawcett and E. G. S. Paige, "Negative differential mobility of electrons in germanium: A Monte Carlo calculation of the distribution function, drift velocity and carrier population in the (111) and (100) minima," $J$. Phys. C, Solid State Phys., vol. 4, no. 13, pp. 1801-1821, Sep. 1971.

[9] S. M. Sze, Physics of Semiconductor Devices. New York: WileyInterscience, 1981.

[10] D. Kazazis, A. Zaslavsky, E. Tutuc, N. A. Bojarczuk, and S. Guha, "Negative differential resistance in ultrathin Ge-on-insulator FETs," Semicond. Sci. Technol., vol. 22, no. 1, pp. S1-S4, Jan. 2007.

[11] K. Uchida, K. Matsuzawa, J. Koga, S.-I. Takagi, and A. Toriumi, "Enhancement of hot-electron generation rate in Schottky source metaloxide-semiconductor field-effect transistors," Appl. Phys. Lett., vol. 76, no. 26, pp. 3992-3994, Jun. 2000.

[12] E. H. Rhoderick and R. H. Williams, Metal-Semiconductor Contacts. Oxford, U.K.: Oxford Science, 1988.

[13] M. Ahmed, "Abrupt negative differential resistance in ungated GaAs FETs," IEEE Trans. Electron Devices, vol. 44, no. 11, pp. 2031-2033, Nov. 1997. 